\title{
Prediabetes and risk for all-cause and cardiovascular mortality based on hypertension status
}

\author{
Yu-Qing Huang", Lin Liu", Jia-Yi Huang, Chao-Lei Chen, Yu-Ling Yu, Kenneth Lo, Ying-Qing Feng \\ Department of Cardiology, Guangdong Cardiovascular Institute, Guangdong Provincial People’s Hospital, Guangdong Academy of Medical \\ Sciences, Guangzhou, China \\ Contributions: (I) Conception and design: YQ Huang, L Liu, K Lo, YQ Feng; (II) Administrative support: YQ Feng; (III) Provision of study materials \\ or patients: YQ Huang, K Lo; (IV) Collection and assembly of data: YQ Huang, L Liu, JY Huang, CL Chen, YL Yu, K Lo; (V) Data analysis and \\ interpretation: YQ Huang, L Liu, K Lo; (VI) Manuscript writing: All authors; (VII) Final approval of manuscript: All authors. \\ \#These authors contributed equally to this work. \\ Correspondence to: Kenneth Lo; Ying-Qing Feng. Department of Cardiology, Guangdong Cardiovascular Institute, Guangdong Provincial People's \\ Hospital, Guangdong Academy of Medical Sciences, No. 106, Zhongshan two Road, Yuexiu District, Guangzhou 510080, China. \\ Email: kenneth_lo@brown.edu; 651792209@qq.com.
}

\begin{abstract}
Background: Current recommendations suggest prediabetes testing for overweight or obese adults with at least one cardiovascular risk factor. However, it is important to understand whether testing should be conducted in adults with hypertension regardless of their cardiovascular risk factors.

Methods: Participants from the 1999-2014 National Health and Nutrition Examination Surveys (NHANES) aged at least 18 years and without diabetes were enrolled in the study. Participants were classified according to their prediabetes and hypertension status and followed up to track cardiovascular and all-cause mortality until December 31, 2015. Cox proportional hazards models were built to estimate the hazard ratios (HRs). Results were stratified by age, sex, ethnicity, body mass index, and cardiovascular disease history.
\end{abstract}

Results: At baseline, 5,011 (14.72\%) out of 34,047 participants had combined prediabetes and hypertension. Prediabetes alone was not associated with all-cause or cardiovascular mortality. Hypertension alone was significantly associated with all-cause (HR: 1.30) and cardiovascular (HR: 1.89) mortality, and combined prediabetes and hypertension were significantly associated with all-cause (HR: 1.37) and cardiovascular (HR: 2.11) mortality. Age and ethnicity were significantly associated with combined prediabetes and hypertension and all-cause mortality, where the association was stronger among people aged <60 years (HR: 1.94 vs. 1.22) and White (HR: 1.50 vs. 1.21).

Conclusions: Prediabetes might elevate the risk of all-cause and cardiovascular mortality among people with hypertension. This relationship is more evident among younger and White individuals.

Keywords: Prediabetes; hypertension; all-cause mortality; cardiovascular mortality

Submitted Feb 01, 2020. Accepted for publication Oct 10, 2020.

doi: 10.21037/atm-20-1155

View this article at: http://dx.doi.org/10.21037/atm-20-1155

\section{Introduction}

Hypertension is one of the most common chronic diseases and is an important modifiable risk factor for cardiovascular metabolic diseases, including diabetes, stroke, and coronary heart disease. It is also a major risk factor for mortality worldwide $(1,2)$. While diabetes has well-established associations with coronary heart disease, ischemic stroke, and hemorrhagic stroke (3), the role of prediabetes in hypertension and cardiovascular disease (CVD) has only recently been receiving increased attention. Prediabetes is defined as an intermediate state between normal glycemia 


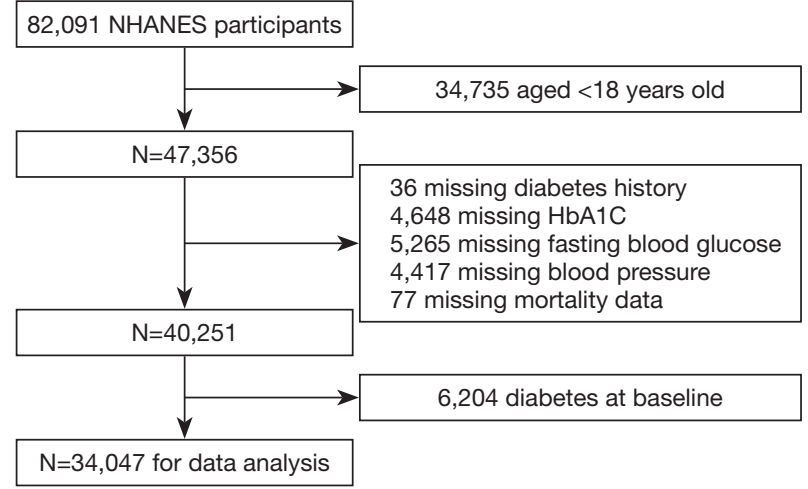

Figure 1 Research flowchart. NHANES, National Health and Nutrition Examination Surveys; HbA1C, hemoglobin A1C.

and diabetes (4), while its prevalence in the United States has greatly increased over the past decades $(5,6)$. Previous studies have found that prediabetes is associated with hypertension (7). The associations between prediabetes and cardiovascular mortality might differ due to hypertension status. Two Chinese cohorts have indicated that participants with combined prediabetes and hypertension had a higher risk for CVD events compared to their counterparts who were free from these conditions $(8,9)$. However, prediabetes alone did not have a significant association with CVD. Similar findings were demonstrated in the Jackson Heart Study, in which prediabetes alone was not associated with an increased risk for CVD events in Black adults without a history of diabetes or CVD at baseline, while an elevated risk was observed in those with both prediabetes and hypertension (10). Given that hypertension is a major risk factor for CVD $(1,2)$, hypertension may cluster with other CVD risk factors including impaired glucose metabolism to influence cardio-metabolic health $(11,12)$. According to the current American Diabetes Association recommendation, testing should be considered for overweight or obese adults with at least one risk factor, such as hypertension, history of CVD, and dyslipidemia (13). Given the detrimental effects of combined prediabetes and hypertension on CVD events, it is important to understand whether testing should be conducted in adults with hypertension regardless of their cardiovascular risk profile. To answer this research question, the association between prediabetes and cardiovascular and all-cause mortality based on hypertension status was analyzed using the data from the National Health and Nutrition Examination Surveys (NHANES).

We present the following article in accordance with the
STROBE reporting checklist (available at http://dx.doi. org/10.21037/atm-20-1155).

\section{Methods}

\section{Study population}

All included participants were from the 1999-2014 NHANES, an ongoing national representative survey of civilian, non-institutionalized population conducted by the Centers for Disease Control and Prevention (14). There were 82,091 participants between 1999 and 2014. Participants with prediabetes and hypertension who aged at least 18 were enrolled. Participants who were younger than 18 , those with missing diabetes history, hemoglobin A1C (HbA1C) or fasting blood glucose (FBG), blood pressure, or mortality history data, and patients diagnosed with diabetes at baseline were excluded. A total of 34,047 participants were included in the final data analysis (Figure 1). The survey protocol was approved by the Institutional Review Board of the Centers for Disease Control and Prevention (Protocol \#98-12, Protocol \#2005-06, Continuation of Protocol \#2005-06, Protocol \#2011-17, Continuation of Protocol \#2011-17), and this study was conducted in accordance with the Declaration of Helsinki (as revised in 2013). All participants have provided written informed consent.

\section{Data collection and covariates}

Demographic characteristics, smoking habits, history of hypertension, diabetes, CVD (any prior diagnosed congestive heart failure, coronary heart disease, angina/ angina pectoris, heart attack, or stroke), or cancer, use of antihypertensive drugs (antiplatelet or anti-dyslipidemia drugs), blood pressure, body mass index (BMI), FBG, total cholesterol (TC), high-density lipoprotein cholesterol (HDL-C), and HbA1C at baseline visit were evaluated through questionnaires or physical examination. Dietary energy was assessed using dietary recall. Diabetes was defined as having FBG $\geq 126 \mathrm{mg} / \mathrm{dL}$, taking hypoglycemic agents, or having $\mathrm{HbA1C} \geq 6.5 \%$. Prediabetes was defined as having FBG at $100-125 \mathrm{mg} / \mathrm{dL}$ or HbA1c between $5.7 \%$ and $6.4 \%$ (13). Hypertension was defined according to the 2018 European Society of Cardiology/European Society of Hypertension (ESC/ESH) Guidelines for the Management of Arterial Hypertension as having blood pressure $\geq$ $140 / 90 \mathrm{mmHg}$ or taking antihypertensive drugs (15). Estimated glomerular filtration rate (eGFR) was calculated 
using Modification of Diet in Renal Disease formula (16).

\section{Outcome definitions}

Participants were followed up until the final outcomes of all-cause and cardiovascular mortality or drop out. Mortality data were extracted from the NHANES public use linked mortality files, which captured the vital status and causes of death in a survey of participants from baseline until December 31, 2015. Cardiovascular mortality was defined by International Classification of Diseases, 10th Edition, Clinical Modification System codes (I00-I09, I11, I13, I20I51) derived from death certificate data.

\section{Statistical analyses}

Baseline characteristics are presented as mean \pm standard deviation (continuous variables) or percentage (categorical variables) as appropriate. One-way analysis of variance, Kruskal-Whallis $\mathrm{H}$ test, and chi-squared test were used for identifying differences between groups. Participants were categorized into four groups as follows: free from prediabetes and hypertension, prediabetes alone, hypertension alone, and combined prediabetes and hypertension. Survival analysis was performed using Kaplan-Meier curves, and logrank test was performed to examine differences between groups. Cox proportional hazards regression models were used to estimate hazard ratios (HRs) and 95\% confidence interval (CI) for all-cause and cardiovascular mortality. Several regressions models were constructed to test for the robustness of findings. Model 1 was not adjusted for any confounders, model 2 was adjusted for age and sex, model 3 was further adjusted for smoking, education, race, BMI, CVD at baseline, cancer at baseline, TC, HDL-C, dietary energy, eGFR, and use of statin or antiplatelet drugs. For subgroup analysis, results were stratified by age $(<60$ or $\geq 60$ years), sex (male or female), BMI ( $<25$ or $\geq 25 \mathrm{~kg} / \mathrm{m}^{2}$ ), CVD at baseline ("yes" or "no"), or race (White or nonWhite) for interaction analysis. A two-sided $\mathrm{P}$ value $<0.05$ was considered statistically significant. All statistical analyses were performed using $\mathrm{R}$ version 3.3.2 (R Foundation for Statistical Computing, Vienna, Austria).

\section{Results}

\section{Participant characteristics}

The baseline characteristics of 34,047 participants are summarized in Table 1. At baseline, 18,451 (54.19\%) participants did not have prediabetes or hypertension, $5,714(16.78 \%)$ had prediabetes alone, 4,871 (14.31\%) had hypertension alone, and 5,011 (14.72\%) had combined prediabetes and hypertension. On average, participants with combined prediabetes and hypertension were older, had higher BMI, received less education, had lower eGFR, were more likely to smoke, had a history of CVD and cancer, and were more likely to use statin, antiplatelet drugs, or antihypertensive drugs compared to the remaining three groups.

\section{Risk for incident CVD and all-cause mortality}

A total of 3,350 (9.84\%) participants died during a median follow-up of 8.4 years, of whom $530(1.56 \%)$ were deceased due to cardiovascular mortality (Table 2). Across all combinations of prediabetes or hypertension status, male participants had significantly higher mortality rates than females (Table 2). The reduction in survival probability for cardiovascular (Figure 2A) and all-cause (Figure 2B) mortality (both $\mathrm{P}$ for log-rank test $<0.001$ ) differed significantly based on prediabetes and hypertension status. The survival rate decreased more rapidly in participants with combined prediabetes and hypertension.

When participants without prediabetes or hypertension were used as a reference (Table 3), prediabetes alone was associated with all-cause (model 1 HR: $2.33,95 \%$ CI: 2.07, 2.63 ) and cardiovascular (model 1 HR: $2.95,95 \%$ CI: 2.03, 4.30) mortality in model 1 . Associations with all-cause (model 2 HR: 0.94, 95\% CI: 0.83, 1.06) and cardiovascular (model 2 HR: 1.01, 95\% CI: 0.69, 1.48) mortality were attenuated after adjusting for age and sex. After full adjustments (model 3), hypertension alone was significantly associated with all-cause (HR: 1.30, 95\% CI: 1.16, 1.45) and cardiovascular (HR: 1.89, 95\% CI: 1.36, 2.61) mortality, while combined prediabetes and hypertension was associated with all-cause (model 3 HR: 1.37, 95\% CI: 1.22, 1.54 ) and cardiovascular (model 3 HR: $2.11,95 \%$ CI: 1.52 , 2.92) mortality.

\section{Subgroup analyses}

According to the subgroup analysis (Table 4), age and race were significantly associated with prediabetes/hypertension status and all-cause mortality (both $\mathrm{P}$ for interaction $<0.05$ ). Participants with hypertension alone (HR: 1.74 vs. 1.17) and combined prediabetes and hypertension had a higher 
Table 1 Baseline characteristics of the participants

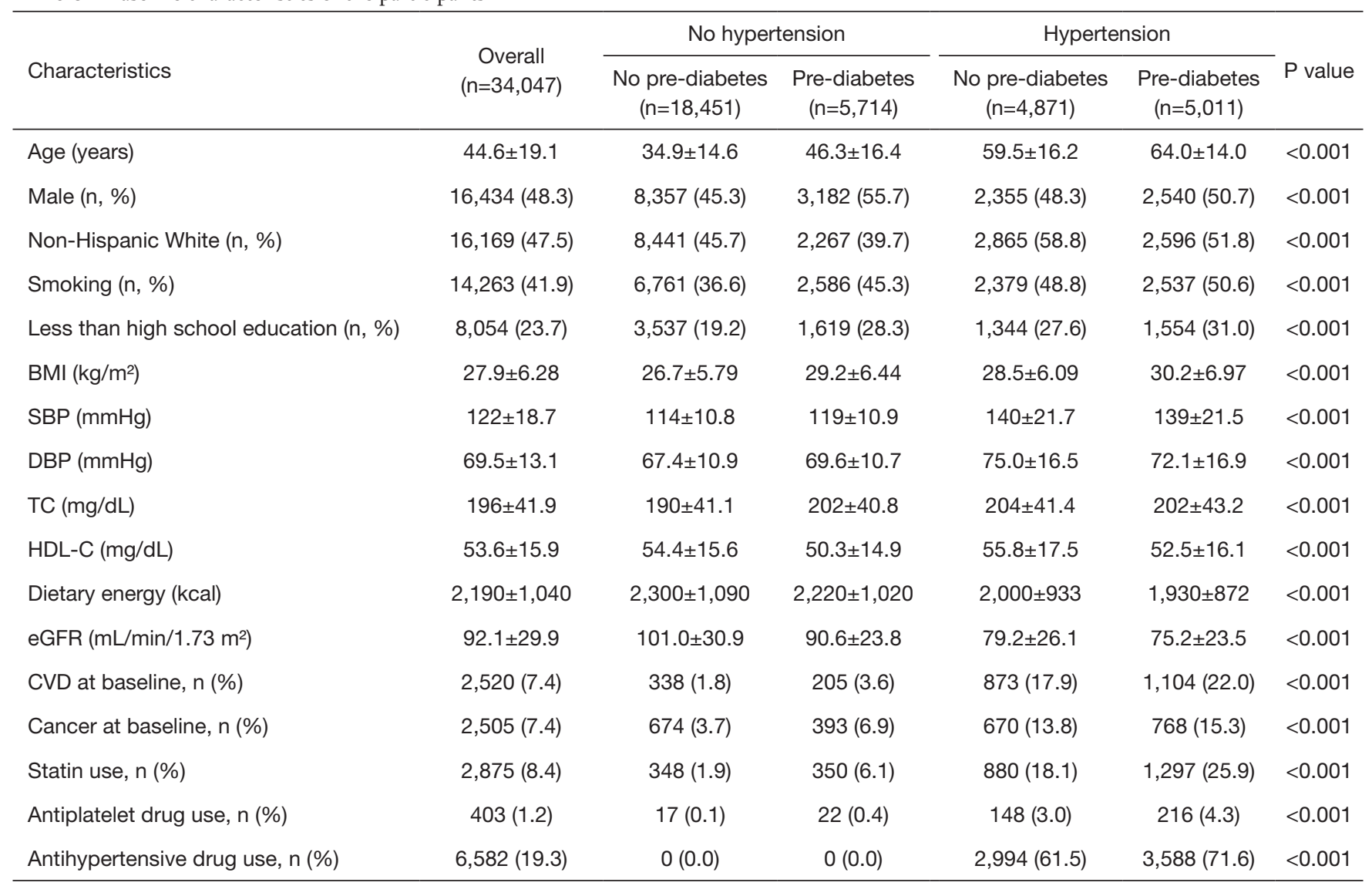

Data are mean \pm standard deviation or percentage. BMI, body mass index; SBP, systolic blood pressure; DBP, diastolic blood pressure; CVD, cardiovascular disease; TC, total cholesterol; HDL-C, high-density lipoprotein cholesterol; eGFR, estimated glomerular filtration rate.

Table 2 Mortality rate by genders, status of pre-diabetes and hypertension

\begin{tabular}{|c|c|c|c|c|c|c|c|c|}
\hline Groups & \multicolumn{4}{|c|}{ All-cause mortality } & \multicolumn{4}{|c|}{ Cardiovascular mortality } \\
\hline $\operatorname{PreDM}(-)$ \& HTN(-) & $747 / 18,451$ & $451(60.4)$ & $296(39.6)$ & $<0.001$ & $66 / 18,451$ & $46(69.7)$ & $20(30.3)$ & $<0.001$ \\
\hline $\operatorname{PreDM}(+)$ \& HTN(-) & $405 / 5,714$ & $272(67.2)$ & $133(32.8)$ & $<0.001$ & $47 / 5,714$ & $32(68.1)$ & 15 (31.9) & 0.116 \\
\hline PreDM(-) \& HTN(+) & $1,083 / 4,871$ & $552(51.0)$ & $531(49.0)$ & $<0.001$ & $190 / 4,871$ & $115(60.5)$ & 75 (39.5) & $<0.001$ \\
\hline Overall & $3,350 / 34,047$ & $1,910(57.0)$ & $1,440(43.0)$ & $<0.001$ & $530 / 34,047$ & $331(62.5)$ & $199(37.5)$ & $<0.001$ \\
\hline
\end{tabular}

DM, diabetes mellitus; HTN, hypertension.

risk of all-cause mortality among those aged $<60$ years (HR: 1.94 vs. 1.22) compared to those aged $\geq 60$ years. Combined prediabetes and hypertension had stronger association with all-cause mortality among White individuals (HR: $1.50 v s$. 1.21) compared to non-White, while hypertension alone was associated with all-cause mortality (HR: 1.43 vs. 1.16). Combined prediabetes and hypertension had a similar association with all-cause mortality when stratified by sex (HR: 1.41 for males $v s .1 .29$ for females), BMI (HR: 1.35 for BMI $\geq 25 \mathrm{~kg} / \mathrm{m}^{2} v s .1 .40$ for $\mathrm{BMI}<25 \mathrm{~kg} / \mathrm{m}^{2}$ ), and CVD 


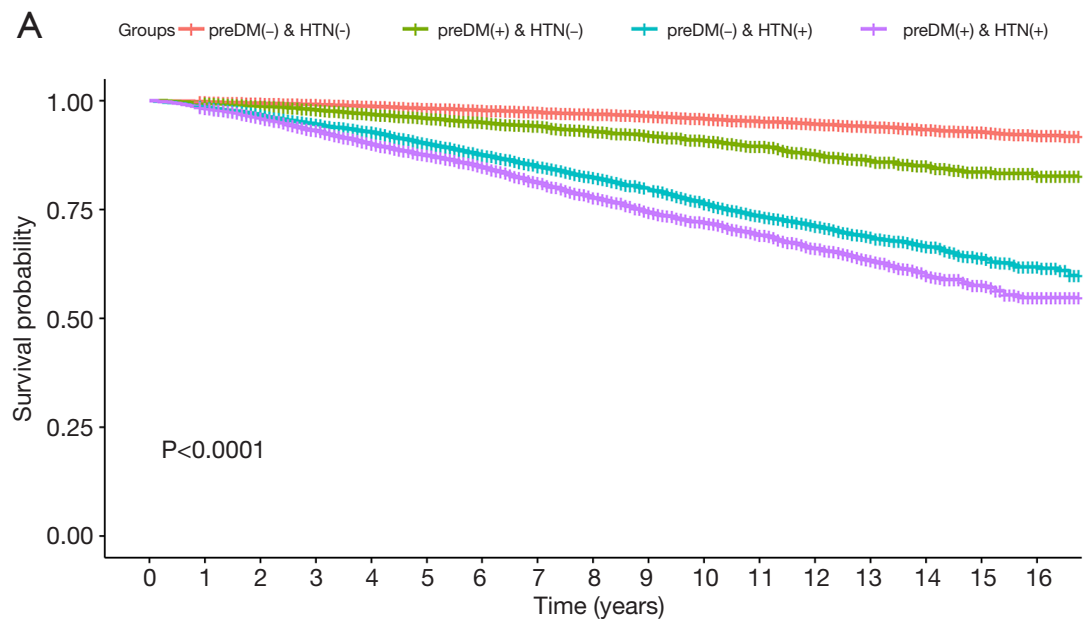

B

$$
\text { Groups }+ \text { preDM(-) \& HTN(-) } \quad+\operatorname{preDM}(+) \& H T N(-) \quad+\operatorname{preDM}(-) \& H T N(+) \quad+\operatorname{preDM}(+) \& H T N(+)
$$

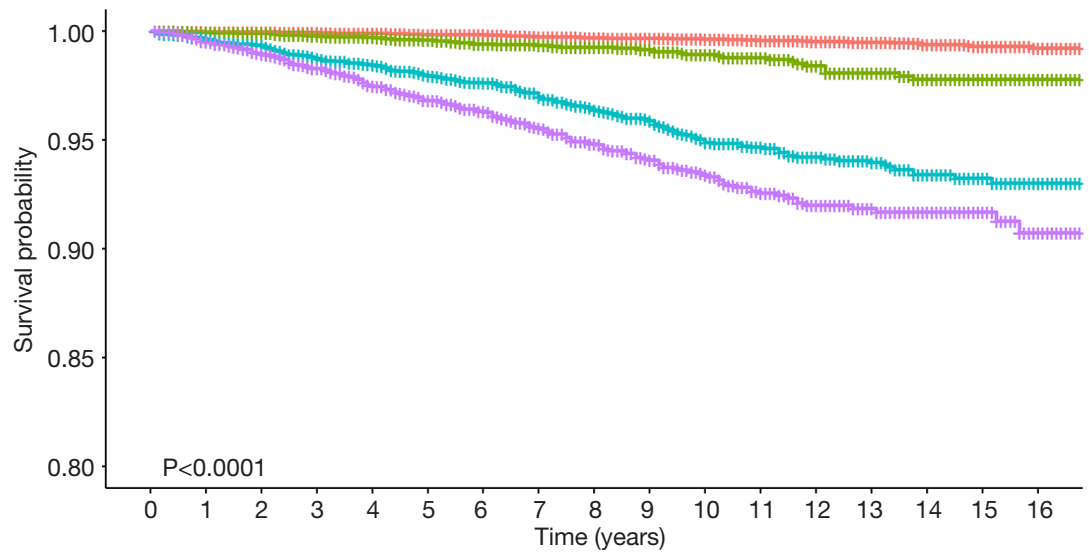

Figure 2 Kaplan-Meier curves for survival in different groups on pre-diabetes and hypertension status. (A) All-cause mortality; (B) cardiovascular mortality. DM, diabetes mellitus; HTN, hypertension.

history (HR: 1.43 for "yes” vs. 1.36 for "no"). When using cardiovascular mortality as the outcome, participants with both prediabetes and hypertension had a higher mortality risk among those aged $<60$ years (2.30 vs. 1.91), female patients (2.28 vs. 1.96), White individuals (2.47 vs. 1.64), and those with $\mathrm{BMI} \geq 25 \mathrm{~kg} / \mathrm{m}^{2}$ (2.09 vs. 2.08) or with CVD at baseline (2.70 vs. 2.05). However, none of the interactions were significant.

\section{Discussion}

In the present study, prediabetes alone was not significantly associated with all-cause or cardiovascular mortality, although a relationship between hypertension and allcause or cardiovascular mortality might exist. According to subgroup analysis, age and race were significantly associated with prediabetes/hypertension status and mortality. The impact of combined prediabetes and hypertension on allcause mortality might be stronger among participants aged $<60$ years and White individuals. Although the interactions were not statistically significant, combined prediabetes and hypertension were associated with all-cause and cardiovascular mortality among younger and White participants.

These findings are consistent with those of some of the previous studies. A cohort study from Iran showed that prediabetes status did not predict any CVD events after adjusting for sex and age (17). The Innovation to Reduce Cardiovascular Complications of Diabetes at the Intersection Study (ARTEMIS) showed that among patients 
Table 3 Associations between pre-diabetes and mortality by hypertension status

\begin{tabular}{|c|c|c|c|c|}
\hline Groups & Case/total & Model $1(\mathrm{~N}=34,047)$ & Model $2(\mathrm{~N}=34,047)$ & Model $3(\mathrm{~N}=29,413)$ \\
\hline PreDM(-) \& HTN(-) & $747 / 18,451$ & Ref. & Ref. & Ref. \\
\hline $\operatorname{PreDM}(+)$ \& HTN(-) & $405 / 5,714$ & $2.33(2.07,2.63)^{\star \star}$ & $0.94(0.83,1.06)$ & $1.05(0.92,1.20)$ \\
\hline PreDM(-) \& HTN(+) & $1083 / 4,871$ & $6.09(5.55,6.68)^{\star \star}$ & $1.24(1.12,1.37)^{\star \star}$ & $1.30(1.16,1.45)^{\star \star}$ \\
\hline $\mathrm{P}$ for trend & & $<0.001$ & $<0.001$ & $<0.001$ \\
\hline \multicolumn{5}{|c|}{ HR for cardiovascular mortality $(95 \% \mathrm{Cl})$} \\
\hline PreDM(-) \& HTN(-) & $66 / 18,451$ & Ref. & Ref. & Ref. \\
\hline PreDM(+) \& HTN(-) & $47 / 5,714$ & $2.95(2.03,4.30)^{\star \star}$ & $1.01(0.69,1.48)$ & $1.24(0.83,1.84)$ \\
\hline$P$ for trend & & $<0.001$ & $<0.001$ & $<0.001$ \\
\hline
\end{tabular}

**, $\mathrm{P}<0.01$. Model 1: unadjusted; Model 2: adjusted for age and gender; Model 3: adjusted for age, gender, smoking, education, race, body mass index, cardiovascular disease at baseline, cancer at baseline, total cholesterol, high-density-lipoprotein cholesterol, dietary energy intake, estimated glomerular filtration rate, the use of statin or antiplatelet drugs. DM, diabetes mellitus; HTN, hypertension; HR, hazard ratio; $\mathrm{Cl}$, confidence interval.

with coronary artery disease, prediabetes was not associated with the risk of cardiovascular and all-cause mortality (18). In contrast, Zhang et al. have demonstrated that impaired glucose tolerance or impaired fasting glucose greatly increased the CVD risk in a pre-hypertensive population, which was free from hypertension and CVD at baseline (19). Moreover, a meta-analysis of 53 prospective cohort studies has demonstrated that prediabetes was significantly associated with an increased risk of cardiovascular and all-cause mortality (20), although this analysis treated hypertension as a confounder instead of examining its combined effect with diabetes. Hypertension is a wellestablished risk factor for CVD events and cardiovascular and all-cause mortality (21-24) and might be significantly associated with CVD and mortality regardless of glucose tolerance status (25). Given the strong relationship between hypertension and CVD, prediabetes is probably linked with CVD only when combined with other metabolic disorders including hypertension (26). The mortality risk for people with prediabetes alone might not be elevated as much as in those with hypertension alone. These explanations were also supported by our findings and those of some previous studies $(8,9)$.

Subgroup analysis revealed that the predictive power of prediabetes or hypertension status for the mortality risk appeared to be stronger among low-risk populations (younger and White participants). One possible explanation was that the high-risk population was already susceptible to a lower survival rate. Therefore, the prediabetes or hypertension status did not substantially elevate the risk. However, hypertension was associated with a higher mortality risk among populations with a lower cardiovascular risk. This risk was even higher when combined with prediabetes.

Currently, several clinical guidelines have provided suggestions for diabetes or prediabetes screening with subtle differences. The American Diabetes Association recommends prediabetes screening for overweight or obese individuals with at least one risk factor, including high risk race (such as African American, Latino, Native American, and Asian American) and history of CVD (13). The ESC recommends diabetes screening for patients with CVD. Individuals with prediabetes but without CVD are not necessarily at elevated cardiovascular risk, but still warrant risk scoring for CVD in the same way as the general population does (27). The National Institute for Health and Clinical Excellence Guidelines on Hypertension also suggested that hypertensive patients should take a blood test to measure HbA1C for potential diabetes (28). In addition, hypertension rarely occurs in isolation and is 
Table 4 Subgroup analysis for the associations between pre-diabetes and mortality by hypertension status

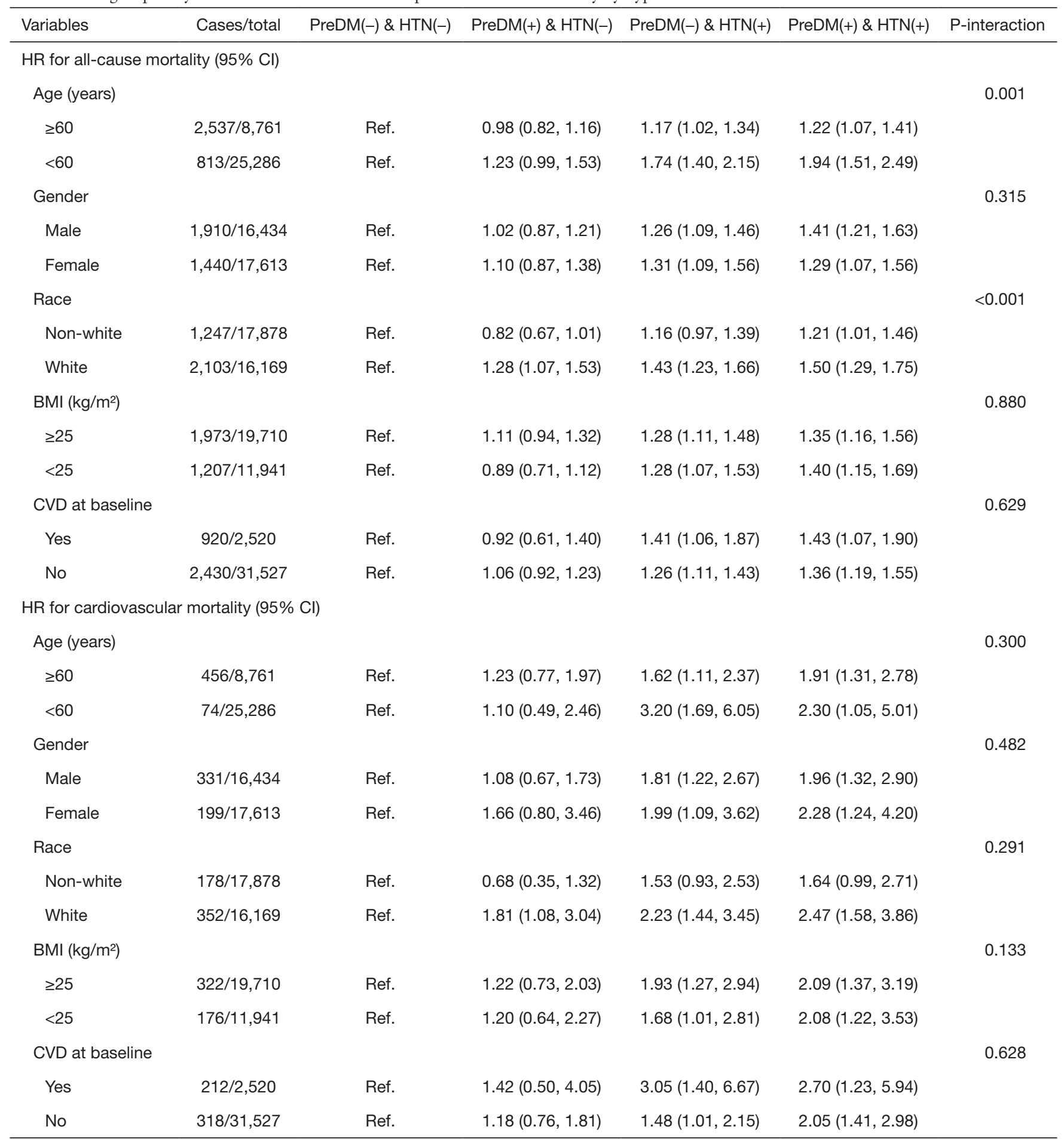

Adjusted for age, gender, smoking, education, race, body mass index, cardiovascular disease at baseline, cancer at baseline, total cholesterol, high-density-lipoprotein cholesterol, energy intake, estimated glomerular filtration rate, the use of statin, antiplatelet drugs. CVD, cardiovascular disease; DM, diabetes mellitus; HTN, hypertension; HDL-C, high-density lipoprotein cholesterol; HR, hazard ratio; Cl, confidence interval. 
often clustered with other CVD risk factors, such as obesity and glucose intolerance. Metabolic risk factor clustering has a multiplicative effect on CVD risk $(11,12)$. According to the $2018 \mathrm{ESC} / \mathrm{ESH}$ guidelines for the management of arterial hypertension, patients with hypertension should have a routine evaluation for multiple cardiovascular risk factors, including fasting glucose and HbA1C (15). However, American College of Cardiology/American Heart Association in 2019 did not recommend prediabetes screening in patients with hypertension (29). Instead, weight and hypertension management were advised for adults with diabetes (29). The present research findings have provided evidence to suggest routine monitoring of glycemic profile in patients with hypertension, while further stratification for high-risk populations may not be necessary. Although prediabetes alone may not predict the risk for a cardiovascular event, it may enhance the detrimental effect of hypertension on cardiovascular health.

Despite providing evidence for hypertension-dependent association between prediabetes and mortality, several study limitations should be noted. First, the present study did not classify prediabetes using glucose tolerance data, which might underestimate the number of participants with prediabetes. Second, considering the cross-sectional nature of NHANES, the proportion of participants that progressed from prediabetes to diabetes was not assessed. Third, few cases of CVD death (1.56\%) have occurred, which might reduce the statistical power. Furthermore, residual confounding occurred due to inaccurate measurement of some adjustment variables such as self-reported lifestyle factors.

To conclude, prediabetes might elevate the risk of all-cause and cardiovascular mortality among people with hypertension. This relationship was more evident among younger participants, males, and non-White individuals. Further studies should consider how the diabetes progression and hypertension status can influence the mortality risk. It is also worthwhile to explore the subpopulation with hypertension for prediabetes screening.

\section{Acknowledgments}

Funding: This work was supported by the Science and Technology Program of Guangzhou (No. 201604020143, No. 201604020018 , No. 201604020186 , and No. 201803040012), the Guangdong Provincial Key Laboratory of Coronary Heart Disease Prevention (No. 2017B030314041), the National Key Research and
Development Program of China (No. 2017YFC1307603, No. 2016YFC1301305) and the Key Area R\&D Program of Guangdong Province (No. 2019B020227005).

\section{Footnote}

Reporting Checklist: The authors have completed the STROBE reporting checklist. Available at http://dx.doi. org/10.21037/atm-20-1155

Conflicts of Interest: All authors have completed the ICMJE uniform disclosure form (available at http://dx.doi. org/10.21037/atm-20-1155). The authors have no conflicts of interest to declare.

Ethical Statement: All the authors are accountable for all aspects of the work in ensuring that questions related to the accuracy or integrity of any part of the work are appropriately investigated and resolved. The survey protocol was approved by the Institutional Review Board of the Centers for Disease Control and Prevention (Protocol \#98-12, Protocol \#2005-06, Continuation of Protocol \#2005-06, Protocol \#2011-17, Continuation of Protocol \#2011-17), and this study was conducted in accordance with the Declaration of Helsinki (as revised in 2013). All participants gave written informed consent.

Open Access Statement: This is an Open Access article distributed in accordance with the Creative Commons Attribution-NonCommercial-NoDerivs 4.0 International License (CC BY-NC-ND 4.0), which permits the noncommercial replication and distribution of the article with the strict proviso that no changes or edits are made and the original work is properly cited (including links to both the formal publication through the relevant DOI and the license). See: https://creativecommons.org/licenses/by-nc-nd/4.0/.

\section{References}

1. Cifu AS, Davis AM. Prevention, Detection, Evaluation, and Management of High Blood Pressure in Adults. JAMA 2017;318:2132-4.

2. Whelton PK, Carey RM, Aronow WS, et al. 2017 ACC/ AHA/AAPA/ABC/ACPM/AGS/APhA/ASH/ASPC/ NMA/PCNA Guideline for the Prevention, Detection, Evaluation, and Management of High Blood Pressure in Adults: A Report of the American College of Cardiology/ American Heart Association Task Force on Clinical 
Practice Guidelines. Hypertension 2018;71:e13-115.

3. Sarwar N, Gao P, Seshasai SR, et al. Diabetes mellitus, fasting blood glucose concentration, and risk of vascular disease: a collaborative meta-analysis of 102 prospective studies. Lancet 2010;375:2215-22.

4. Nathan DM, Davidson MB, DeFronzo RA, et al. Impaired fasting glucose and impaired glucose tolerance: implications for care. Diabetes Care 2007;30:753-9.

5. Bullard KM, Saydah SH, Imperatore G, et al. Secular changes in U.S. Prediabetes prevalence defined by hemoglobin A1c and fasting plasma glucose: National Health and Nutrition Examination Surveys, 1999-2010. Diabetes Care 2013;36:2286-93.

6. Menke A, Casagrande S, Geiss L, et al. Prevalence of and Trends in Diabetes Among Adults in the United States, 1988-2012. JAMA 2015;314:1021-9.

7. Jung JY, Oh CM, Choi JM, et al. Long-Term Risk of Hypertension in Normoglycemia and Prediabetes, and Their Relation to the Change of Glycemic State. Am J Hypertens 2018;31:1042-8.

8. Qiu M, Shen W, Song X, et al. Effects of prediabetes mellitus alone or plus hypertension on subsequent occurrence of cardiovascular disease and diabetes mellitus: longitudinal study. Hypertension 2015;65:525-30.

9. Liu HH, Cao YX, Li S, et al. Impacts of Prediabetes Mellitus Alone or Plus Hypertension on the Coronary Severity and Cardiovascular Outcomes. Hypertension 2018;71:1039-46.

10. Hubbard D, Colantonio LD, Tanner RM, et al. Prediabetes and Risk for Cardiovascular Disease by Hypertension Status in Black Adults: The Jackson Heart Study. Diabetes Care 2019;42:2322-9.

11. Collins DR, Tompson AC, Onakpoya IJ, et al. Global cardiovascular risk assessment in the primary prevention of cardiovascular disease in adults: systematic review of systematic reviews. BMJ Open 2017;7:e13650.

12. Cannon CP. Cardiovascular disease and modifiable cardiometabolic risk factors. Clin Cornerstone 2007;8:11-28.

13. American Diabetes Association. Classification and Diagnosis of Diabetes: Standards of Medical Care in Diabetes-2019. Diabetes Care 2019;42:S13-28.

14. Fain JA. NHANES. Diabetes Educ 2017;43:151.

15. Williams B, Mancia G, Spiering W, et al. 2018 ESC/ESH Guidelines for the management of arterial hypertension. Eur Heart J 2018;39:3021-104.

16. National Kidney Foundation. K/DOQI clinical practice guidelines for chronic kidney disease: evaluation, classification, and stratification. Am J Kidney Dis 2002;39:S1-S266.

17. Khosravi A, Gharipour M, Nezafati P, et al. Prehypertension, pre-diabetes or both: which is best at predicting cardiovascular events in the long term? J Hum Hypertens 2017;31:382-7.

18. Kiviniemi AM, Lepojarvi ES, Tulppo MP, et al. Prediabetes and Risk for Cardiac Death Among Patients with Coronary Artery Disease: The ARTEMIS Study. Diabetes Care 2019;42:1319-25.

19. Zhang Y, Lee ET, Devereux RB, et al. Prehypertension, diabetes, and cardiovascular disease risk in a populationbased sample: the Strong Heart Study. Hypertension 2006;47:410-4.

20. Huang Y, Cai X, Mai W, et al. Association between prediabetes and risk of cardiovascular disease and allcause mortality: systematic review and meta-analysis. BMJ 2016;355:i5953.

21. Yano Y, Rakugi H, Bakris GL, et al. On-Treatment Blood Pressure and Cardiovascular Outcomes in Older Adults with Isolated Systolic Hypertension. Hypertension 2017;69:220-7.

22. Zhao L, Song Y, Dong P, et al. Brachial pulse pressure and cardiovascular or all-cause mortality in the general population: a meta-analysis of prospective observational studies. J Clin Hypertens (Greenwich) 2014;16:678-85.

23. Wu CY, Hu HY, Chou YJ, et al. High Blood Pressure and All-Cause and Cardiovascular Disease Mortalities in Community-Dwelling Older Adults. Medicine (Baltimore) 2015;94:e2160.

24. Redon J, Tellez-Plaza M, Orozco-Beltran D, et al. Impact of hypertension on mortality and cardiovascular disease burden in patients with cardiovascular risk factors from a general practice setting: the ESCARVAL-risk study. J Hypertens 2016;34:1075-83.

25. Hajebrahimi MA, Akbarpour S, Eslami A, et al. Different combinations of glucose tolerance and blood pressure status and incident cardiovascular disease and all-cause mortality events. J Hum Hypertens 2017;31:744-9.

26. Liu J, Grundy SM, Wang W, et al. Ten-year risk of cardiovascular incidence related to diabetes, prediabetes, and the metabolic syndrome. Am Heart J 2007;153:552-8.

27. Cosentino F, Grant PJ, Aboyans V, et al. 2019 ESC Guidelines on diabetes, pre-diabetes, and cardiovascular diseases developed in collaboration with the EASD. Eur Heart J 2020;41:255-323.

28. Krause T, Lovibond K, Caulfield M, et al. Management of hypertension: summary of NICE guidance. BMJ 
Page 10 of 10

2011;343:d4891.

29. Arnett DK, Blumenthal RS, Albert MA, et al. 2019 ACC/AHA Guideline on the Primary Prevention of Cardiovascular Disease: Executive Summary: A Report
Huang et al. Prediabetes and hypertension status with mortality

of the American College of Cardiology/American Heart Association Task Force on Clinical Practice Guidelines. J Am Coll Cardiol 2019;74:1376-414.

Cite this article as: Huang YQ, Liu L, Huang JY, Chen CL, Yu YL, Lo K, Feng YQ. Prediabetes and risk for all-cause and cardiovascular mortality based on hypertension status. Ann Transl Med 2020;8(23):1580. doi: 10.21037/atm-20-1155 